\title{
Two-photon imaging with thermal light
}

\author{
Alejandra Valencia, Giuliano Scarcelli, Milena D'Angelo and Yanhua Shih \\ Department of Physics, University of Maryland, Baltimore County, \\ Baltimore, Maryland 21250
}

We report the first experimental demonstration of twophoton imaging with a pseudo-thermal source. Similarly to the case of entangled states, a two-photon Gaussian thin lens equation is observed, indicating EPR type correlation in position. We introduce the concepts of two-photon coherent and two-photon incoherent imaging. The differences between the entangled and the thermal cases are explained in terms of these concepts.

Two-photon imaging has been the subject of massive studies since its first demonstration ten years ago $[1,2]$. In a general sense two-photon imaging involves a joint detection of two photons at distant space-time points. The radiation from a source is split into two separate optical paths; an object (aperture) is placed in one of the paths, but the spatial information of the object is recuperated in a nonlocal fashion by means of the second order correlation measurement. The effect of two-photon imaging has been brought to the general attention by an experiment that exploited the quantum entanglement nature of a photon pair generated via Spontaneous Parametric Down Conversion (SPDC) [2]. In that experiment the signal and idler radiation of SPDC were sent to two distant detectors. An aperture and an imaging lens were placed in the signal arm of the optical setup just before a bucket detector; there was no optical element in the idler arm; however, by scanning the idler detector in the plane defined by a two-photon Gaussian thin lens equation, a sharp and magnified image of the aperture was obtained in the coincidence counts, even though the single counting rates of both detectors were fairly constant during the scanning. This historical experiment was named ghost imaging due to the fact that the image of the object is formed by photons that never actually pass through the aperture. Further investigations on entangled photons brought to the development of a new field, named twophoton geometric optics [3].

Recently it has been argued that classically correlated light might mimic some features of the entangled photon pairs in coincidence imaging setups [4]. Notice that the possibility of simulating the two-photon imaging features of entangled states with classical sources was not ruled out by the authors of the original ghost imaging experiment [2]. Both the theoretical work of Abourraddy et.al. [5] and the experimental investigation of Bennink et.al. [4] stimulated a very interesting debate about the role of entanglement in two-photon coincidence imaging [6-11]. In particular Bennink et al. [4] have experimentally demonstrated the possibility of performing "farfield" coincidence imaging, i.e., measuring momentum correlation only, with pairs of photons (pulses) classically correlated in momentum. Very recently Gatti et.al. [12] have proposed thermal (or pseudo-thermal) radiation as a classical source to perform "near-field" coincidence imaging, which measures position correlation (at least partially), in a specific optical setup. Since then a great deal of attention on the subject has been induced [13].

In this paper we wish to present the first experimental demonstration of two-photon ghost imaging with thermal radiation. In particular, we show that a thermal source is able to simulate one of the main features of entangled two-photon imaging: the two-photon Gaussian thin lens equation, i.e., the EPR type correlation in position is partially observable. The expression partially accounts for the reduced visibility of thermal two-photon images (50\% constant background). Furthermore, we introduce the concepts of two-photon incoherent and two-photon coherent imaging to explain the fundamental differences between thermal and entangled two-photon imaging. Notice that the thermal ghost image presented here is the first authentic simulation of the quantum ghost image [2]. In fact, no two-photon thin lens equation, which is a symbol of position correlation, can be found by replacing SPDC with a source of photons classically correlated in momentum [4]. Thermal sources provide an ideal comparison to quantum ghost imaging effects for a more complete understanding of two-photon, or two-particle physics.

The behavior of entangled two-photon systems has been well studied $[14,15]$. It is possible to establish an analogy between classical optics and entangled twophoton optics: the two-photon probability amplitude plays in entangled two-photon processes the same role that the complex amplitude of the electric field plays in classical optics; the role played by the intensity of the electromagnetic field in classical optics is played by the rate of coincidence counts, and therefore by the time integrated second-order correlation function in entangled two-photon processes. For both thermal source and SPDC, the two-photon thin lens equation is the result of the coupling of two-photon probability amplitudes. However, for an SPDC source, one pair of photons contains all the two-photon probability amplitudes that generate the ghost image; we define the result as two-photon coherent image. For a thermal source, instead, the various two-photon probability amplitudes come from the ensemble of many independent pairs that generates the ghost image; thus, we define it as two-photon incoherent image.

The experimental setup is shown in Fig. 1. After the pseudo-thermal source [16,17], a non-polarizing beam splitter $(B S)$ splits the radiation in two distinct optical paths. In the reflected arm an object, with transmission function $T\left(\overrightarrow{x_{1}}\right)$, is placed at a distance $d_{A}$ from the $B S$ 
and a bucket detector $\left(D_{1}\right)$ is just behind the object. In the transmitted arm an imaging lens, with focal length $f$, is placed at a distance $d_{B}$ from the $B S$, and a multimode optical fiber (then connected to detector $D_{2}$ ) scans the transverse plane at a distance $d_{B}^{\prime}$ from the lens. The output pulses from the two single photon counting detectors are then sent to an electronic coincidence circuit, to measure the rate of coincidence counts.

The rate of coincidence counts is governed by the second order Glauber correlation function [18]:

$$
\begin{aligned}
& G^{(2)}\left(t_{1}, \vec{r}_{1} ; t_{2}, \vec{r}_{2}\right) \equiv \\
& \left\langle E_{1}^{(-)}\left(t_{1}, \vec{r}_{1}\right) E_{2}^{(-)}\left(t_{2}, \vec{r}_{2}\right) E_{2}^{(+)}\left(t_{2}, \vec{r}_{2}\right) E_{1}^{(+)}\left(t_{1}, \vec{r}_{1}\right)\right\rangle .
\end{aligned}
$$

where $E^{(-)}$and $E^{(+)}$are the negative-frequency and the positive-frequency field operators describing the detection events at the space-time locations $\left(\overrightarrow{r_{1}}, t_{1}\right)$ and $\left(\overrightarrow{r_{2}}, t_{2}\right)$. The transverse second order correlation function (at equal times) for a thermal source is given by [19]:

$$
\begin{aligned}
G_{\text {thermal }}^{(2)}\left(\vec{x}_{1} ; \vec{x}_{2}\right) & \propto \sum_{\vec{q}}\left|g_{1}\left(\vec{x}_{1}, \vec{q}\right)\right|^{2} \sum_{\vec{q}^{\prime}}\left|g_{2}\left(\vec{x}_{2}, \vec{q}^{\prime}\right)\right|^{2} \\
& +\left|\sum_{\vec{q}} g_{1}^{*}\left(\vec{x}_{1}, \vec{q}\right) g_{2}\left(\vec{x}_{2}, \vec{q}\right)\right|^{2}
\end{aligned}
$$

where $\overrightarrow{x_{i}}$ is the transverse position of detector $D_{i}, \vec{q}$ and $\overrightarrow{q^{\prime}}$ are the transverse components of the momentum vectors and $g_{i}\left(\vec{x}_{i} ; \vec{q}\right)$ is the Green's function associated with the propagation of the field from the source to the $i^{\text {th }}$ detector [20]. Notice that there are two differences with respect to the SPDC case: (1) the presence of a background noise (first term of Eq. 2), which does not exist for SPDC; (2) the possibility of writing the second term of Eq. 2 as a product of first order correlation functions, $G_{12}^{(1)} G_{21}^{(1)}$ while the second order correlation function for SPDC, cannot be reduced to a mere product of first order correlation functions in any format.

For the setup of Fig. 1, it can be shown that for any values of the distances $d_{A}, d_{B}$, and $d_{B}^{\prime}$ which obey the equation:

$$
\frac{1}{d_{B}-d_{A}}+\frac{1}{d_{B}^{\prime}}=\frac{1}{f}
$$

Eq. 2 can be simplified as [19]:

$$
G_{t o t}^{(2)}\left(\vec{x}_{2}\right) \propto N+\left|T\left(\frac{d_{A}-d_{B}}{d_{B}^{\prime}} \vec{x}_{2}\right)\right|^{2},
$$

where $T\left(\frac{d_{A}-d_{B}}{d_{B}^{\prime}} \vec{x}_{2}\right)$ is the object transmission function $\left(T\left(\overrightarrow{x_{1}}\right)\right)$ reproduced on the $D_{2}$ plane. We can then conclude that a thermal source allows reproducing in coincidence measurements the ghost image of an object, similarly to the SPDC case, except for a constant background noise. The constant background noise, first term in Eq. 4, is proportional to the total transmittance of the object and, therefore, can be simplified to $\mathrm{N}$, the number of transparent features in the object plane [19]. Eq. 3 can clearly be interpreted as a two-photon Gaussian thin lens equation, in which the object distance is $s_{o}=d_{B}-d_{A}$ and the image distance is $s_{i}=d_{B}^{\prime}$ (see Fig. 2). We expect to observe an inverted image magnified by a factor of $M=s_{i} / s_{o}$. Notice that when only one slit is inserted in the object plane, the maximum achievable visibility is $33 \%$. The visibility, however, is expected to drop when the number of transparent features increases.

The discovery of general laws in physics allows considering pictorial representations which may turn out to be powerful but still simple predictive tools. In this sense Klyshko's pictures for SPDC imaging experiments $[21,2]$ are exemplar. The results presented in Eq. 3 and Eq. 4 also offer the possibility of considering a generalized Klyshko's representation; nevertheless, the presence of two terms in Eq. 4 makes the thermal Klyshko's picture more complex. For simplicity, in Fig. 2 we only picture the second term of Eq. 4, which is the one that produces the ghost image. Since the source is chaotic, each atom of the source randomly emits photons with all possible values of momentum. A photon with momentum $\vec{q}$ arriving at $\vec{x}_{1}$ can produce a coincidence count with any other photon emitted by the source and arriving at $\vec{x}_{2}$. These coincidences result in a background noise that does not lead to the production of an image. This part of the physical process is not reflected in Fig. 2. Notice that, similarly to the SPDC case, the point-to-point correspondence between object and image plane is the result of the addition of two-photon probability amplitudes. It is important to point out however a remarkable difference between the thermal and the SPDC case. In the Klyshko's picture for SPDC each pair of lines corresponds to a two-photon probability amplitude and all of them are associated to one pair, hence we define the image twophoton coherent. In the diagram for the thermal case each pair of lines represents the term $g_{1}^{*}\left(\vec{x}_{1}, \vec{q}\right) g_{2}\left(\vec{x}_{2}, \vec{q}\right)$ and the various lines are associated to different pairs of photons. It is apparent then that the thin lens equation is the result of the coupling $g_{1}^{*}\left(\vec{x}_{1}, \vec{q}\right) g_{2}\left(\vec{x}_{2}, \vec{q}\right)$, but the image is generated by the ensemble of many independent pairs, leading us to the definition of two-photon incoherent image. The diagram of Fig. 2 also shows that while in the Klyshko's picture for SPDC the source behaves as an ordinary mirror, here the thermal source behaves as a phase conjugated mirror; this is due to the definition of the object distance $s_{o}=d_{B}-d_{A}$. This interpretation indicates the presence of a pseudo-object in the plane $\sigma$, as shown in Fig. 2; scanning this plane the pseudo-object was actually observed (see also Fig. 3).

Our first experimental measurement was aimed to verify the existence of a point-to-point correspondence between object and image plane, as expected by the existence of a thin lens equation. The setup is the same as that of Fig. 1, but we used the $60 \mu \mathrm{m}$-diameter input aperture of an optical fiber as the object (whose output was then coupled to detector $D_{1}$ ). As a preliminary measurement we studied the temporal second order cor- 
relation function. An optimized coincidence time window was chosen accordingly. For the actual spatial correlation measurement, we collected three sets of data for three different positions of the fiber in the object plane; in every measurement we kept the position of the fiber fixed and scanned detector $D_{2}$ in the transverse direction. The results are shown in Fig. 3: any shift of the fiber in the object plane causes a shift of the correlation function in the opposite direction, in analogy to standard geometrical optics. In particular Fig. 3 shows that shifting the fiber in the object plane by $2 \mathrm{~mm}$ the correlation function shifts by $4.3 \mathrm{~mm}$. Hence, the magnification in the imaging plane is $M_{\text {meas }}=2.15$, which is very close to the expected value $\left(M_{\text {expect }}=2.16\right)$. The achieved visibility is $26 \%$. The results shown in Fig. 3 clearly show the point-to-point correspondence between object and imaging plane in agreement with Eq. 3 and Eq. 4.

Then we placed a double slit in the object plane (center to center separation $1 \mathrm{~mm}$, slit width $0.2 \mathrm{~mm}$ ) and repeated the measurement. The results are shown in Fig. 4. As expected, the single counts are flat, while the coincidence counts reproduce the magnified image of the double slit. The visibility drops to about $12 \%$.

The experimental data show that the visibility of the two-photon image drops with the number of transparent features in the object plane, as predicted in Eq. 4. This effect is readily understood by inspecting Fig. 3: for each feature in the object plane, the whole image plane shows besides the expected image a non-negligible noise level. Hence, if in the object plane there are simultaneously three features, in the image plane we will observe the addition of the three graphs of Fig. 3, as predicted by Eq. 4 . This clearly indicates that the background noise increases with the number of transparent features to image at the expenses of the visibility.

The physics behind quantum ghost image and thermal ghost image can be understood as follows: in both cases the image is the result of the addition of two-photon probability amplitudes nevertheless such probability amplitudes have very different origins in the two cases. In SPDC, a signal-idler pair is described by a pure state given by the superposition of an infinite number of twophoton probability amplitudes. On the other hand, thermal sources are modelled as an incoherent statistical mixture of many pairs of photons; the various two-photon probability amplitudes are provided by the entire ensemble of photon pairs. The different models come from the different processes involved in the generation of SPDC and thermal radiation. SPDC comes from a nonlinear coherent interaction and consequently it is impossible to identify the birth place of a photon pair. This is why each photon pair contains simultaneously all the possible transverse momenta necessary for imaging. On the other hand, for a thermal source each photon is generated from a random excitation of independent atoms. This is in line with the interpretation of the two Klyshko's pictures given above and clarifies what we mean by two-photon coherent and two-photon incoherent imaging.
In conclusion, we have presented the first experimental demonstration of two-photon ghost imaging with thermal-like sources. For the first time a two-photon Gaussian thin lens equation is found for non-entangled sources, indicating the existence of, at least partial, EPR type correlation in position. We have also introduced the concepts of two-photon coherent and two-photon incoherent imaging to describe the different physics behind entangled and thermal ghost imaging. The already poor visibility of the thermal ghost image (max 33\%), drops rapidly for complicated apertures; therefore, practical applications of thermal ghost imaging rely on the development of a detection scheme which is insensitive to the background noise. Such detection scheme is now under development in our laboratory.

The authors would like to thank S. Thanvanthri, J. Wen and M.H. Rubin for everyday helpful discussions. Y.H.S. specially thanks S.Y. Zhu for stimulating discussions and encouragement. This research was supported in part by NSF, ONR and NASA-CASPR program.

[1] A.V. Belinskii and D.N. Klyshko, Sov. Phys. JETP 78, 259 (1994).

[2] T.B. Pittman, Y.H. Shih, D.V. Strekalov, and A.V. Sergienko, Phys. Rev A 52 R3429 (1995).

[3] T.B. Pittman, D.V. Strekalov, D.N. Klyshko, M.H. Rubin, A.V. Sergienko, and Y.H. Shih, Phys. Rev. A 53, 2804 (1996).

[4] R.S. Bennink, S.J. Bentley, and R.W. Boyd, Phys. Rev. Lett 89, 113601 (2002).

[5] A.F. Abouraddy, B.E.A. Saleh, A.V. Sergienko and M.C. Teich, Phys. Rev. Lett. 87, 123602 (2001)

[6] A.F. Abouraddy, B.E.A. Saleh, A.V. Sergienko and M.C. Teich, J. Opt. Soc. Am. B 19, 1174 (2002)

[7] A. Gatti, E. Brambilla, and L.A. Lugiato, Phys. Rev. Lett. 90, 133603 (2003)

[8] R.S. Bennink, S.J. Bentley, R.W. Boyd, and J.C. Howell, Phys. Rev. Lett 92, 033601 (2004).

[9] M.H. Rubin, quant-ph/0303188.

[10] M. D'Angelo, Y.H. Kim, S.P. Kulik, and Y. Shih, Phys. Rev. Lett. 92, 233601 (2004)

[11] G.Bjork, J.Soderholm, and L.L. Sanchez-Soto, J.Opt. B 6, 478 (2004)

[12] A.Gatti, E. Brambilla, M.Bache and L.A. Lugiato, Phys. Rev. A 70, 013802, (2004).

[13] Y.J. Cai and S.Y. Zhu, Opt. Lett. 29, 2716 (2004); J.Bogdansky, G.Bjork, A. Karlsson quant-ph/0407127; K. Wang and D.Z. Cao, Phys. Rev. A. 70, 041801(R) (2004); D.Magatti, F.Ferri, A. Gatti, M. Bache, E. Brambilla, L.A. Lugiato, quant-ph/0408021.

[14] A.V. Belinsky and D.N. Klyshko, Laser Physics 4, 663 (1994)

[15] Y. Shih, IEEE J. of Selected Topics in Quantum Electronics, 9, 1455 (2003) 
[16] W. Martienssen and E. Spiller, Am. J. Phys. 32, 919 (1964).

[17] G. Scarcelli, A.Valencia and Y. Shih, Phys. Rev. A 70, 051802(R) (2004).

[18] R.J. Glauber, Phys. Rev. 130, 2529 (1963); 131, 2766 (1963).

[19] A. Valencia, G. Scarcelli, M. D'Angelo, and Y. Shih, subm. to Phys. Rev. A (2004)

[20] M. H. Rubin, Phys. Rev. A 54, 5349, (1996).

[21] D.N. Klyshko, Photons and Nonlinear Optics (Gordon \& Breach, New York,1988).

FIG. 1. Experimental setup. Source diameter $\sim 200 \mu m$; $a=125 \mathrm{~mm} ; d_{A}=88 \mathrm{~mm} ; d_{B}=212 \mathrm{~mm} ; d_{B}^{\prime}=268.5 \mathrm{~mm}$ $f=85 \mathrm{~mm}$; fiber tip diameter $=60 \mu$.

FIG. 2. Conceptual unfolded version of the optical setup. Object plane and image plane obey a two-photon gaussian thin lens equation and are defined by $s_{o}=d_{B}-d_{A}$ and $s_{i}=d_{B}^{\prime}$, respectively. In terms of Klyshko's picture, the thermal source acts as a phase conjugated mirror forming a pseudo-object in the $\sigma$ plane.

FIG. 3. Normalized second order correlation function vs. position of $D_{2}$. The measurement shows the point-to-point correspondence between object and image planes. a) Tip of the fiber in the object plane located in the position indicated by the square. b) Tip of the fiber in the object plane located in the position indicated by the circle (central position). c) Tip of the fiber in the object plane located in the position indicated by the triangle.

FIG. 4. Single and coincidence counts vs. transverse position of $D_{2}$ in the image plane $\left(x_{2}\right)$. Single counts of both $D_{1}$ (hollow circles) and $D_{2}$ (full circles) are flat. The coincidence counts (solid line with circles) show a magnified image of the object.

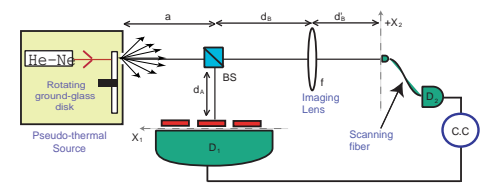

Figure 1. Alejandra Valencia, Giuliano Scarcelli, Milena D'Angelo, and Yanhua Shih.

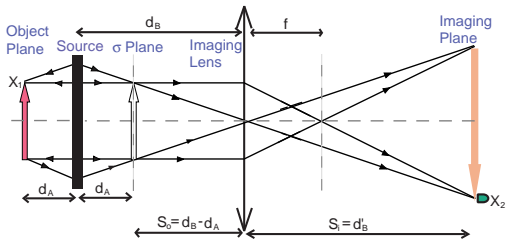

Figure 2. Alejandra Valencia, Giuliano Scarcelli, Milena D'Angelo, and Yanhua Shih.
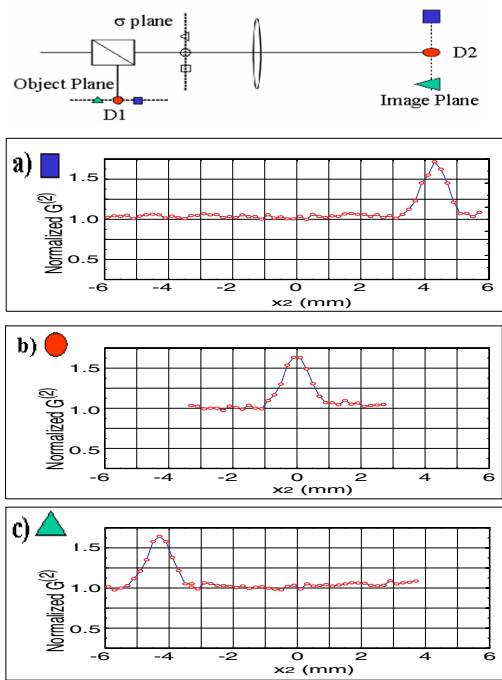

Figure 3. Alejandra Valencia, Giuliano Scarcelli, Milena D'Angelo, and Yanhua Shih.

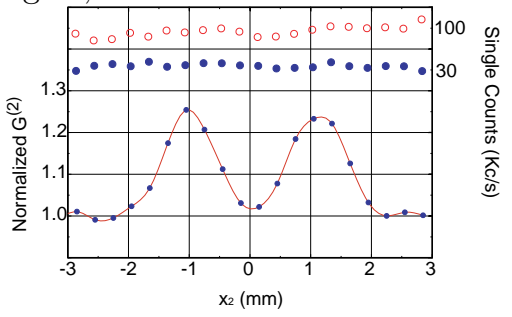

Figure 4. Alejandra Valencia, Giuliano Scarcelli, Milena D'Angelo, and Yanhua Shih. 